\title{
Modeling and simulation of Caenorhabditis elegans chemotaxis in response to a dynamic engineered bacteria
}

\author{
Divya A Pandya, Christopher A Blanar, Robert P Smith, Evan C Haskell \\ Division of Math, Science, and Technology \\ Farquhar College of Arts and Sciences \\ Nova Southeastern University \\ Ft. Lauderdale, FL 33314 USA \\ Email: haskell@nova.edu
}

\begin{abstract}
KEYWORDS
nematodes, agent-based modeling, chemoattractants, learning, Caenorhabditis elegans, periodic environment, movement ecology
\end{abstract}

\begin{abstract}
Parasitic helminthes remain important causative agents of human, plant and animal diseases. Helminthes seek out food sources and navigate toward potential hosts using olfaction of simple chemical cues in a process called chemoattraction. While several studies have examined how nematodes, including Caenorhabditis elegans, behave in response to a chemoattractant, how the characteristics of the chemoattractant affect worm behavior has yet to explored. In this manuscript, we develop a mathematical model to examine how characteristics of common chemoattractants affect movement and behavior in the model nematode $C$. elegans. Specifically, we model a scenario where a toxic, engineered bacteria designed to express a chemoattractant influences the behavior of a population of worms. Through the model we observe that, under static conditions, the diffusion rate of the chemoattractant is critical in influencing choice of $C$. elegans. Here, the higher diffusion rate, the more the worms are attracted to the chemoattractant. We then show that if the worms learn that the chemoattractant is associated with toxicity, choice index is counterintuitively more strongly reduced with increasing diffusion rate. Finally, our model predicts a tradeoff between pulse period and attractant strength when the chemoattractant is dynamically pulsed in the environment. Our results reveal unique tradeoffs that govern chemoattraction in worms and may have implications in designing novel strategies for preventing or treating infections with parasitic worms.
\end{abstract}

\section{INTRODUCTION}

Parasitic helminthes remain important causative agents of human disease (e.g., (De Silva et al., 2003)), particularly in developing nations (Hotez et al., 2008). Parasitic nematodes such as hookworms are significant problems in many parts of the world, causing morbidity and exacting a cruel toll of hardship on affected human populations (Brooker et al., 2004). Helminthes, including the nematodes, possess a remarkable array of adaptations to facilitate the infection of and subsequent establishment within their host(s). Whereas trophically transmitted helminthes rely on ingestion by their host(s) (Lafferty, 1999) and vector-borne parasites are transmitted by arthropods (Chaisson and Hallem, 2012), many helminthes must actively seek out their host in the environment by moving through water or soils (Sciacca et al., 2002). Furthermore, once inside the host, helminthes are able to navigate to preferred infection sites within the host (Grabe and Haas, 2004). Mounting evidence suggests that movements toward and within the host are guided by olfaction using simple chemical cues, or chemoattractants (Chaisson and Hallem, 2012; Haas, 2003). Remarkably, several nematodes are able to selectively seek out preferred host species based on chemoattractants demonstrating high specificity in signal integration and movement (e.g,(Chaisson and Hallem, 2012; Safer et al., 2007; Rae et al., 2009)). Several helminthes, particularly nematodes, will also use chemoattraction to find food sources, such as bacteria, during their larval stage prior to infecting their definitive host (Zuckerman and Jansson, 1984).

Despite evidence demonstrating that a wide range of chemoattractants influence helminth behavior and movement, little is known regarding how the properties of the chemoattractant itself govern the attraction process. An understanding of how chemoattractant properties affect the movement of helminthes may be critical in developing new strategies to preventing human infections and developing alternative strategies to prevent crop destruction (Chaisson and Hallem, 2012).

Animal movements such as that of Caenorhabditis elegans, represent a complex spatio-temporal phenomena driven by the interaction of behavioral processes and external environmental factors. Agent based modeling $(\mathrm{ABM})$ provides a framework for integrative bottom up modeling for simulation of complex adaptive movement dynamics (Tang and Bennett, 2010; Railsback and Grimm, 2011). To describe mechanisms underlying movement, four basic components are needed: internal state, motion capacity, navigation capacity, and external factors (Nathan, 2008). Thus ABM is a framework well suited for the study of individual animal movement and exploration of factors related to emergent population level decision patterns.

In this manuscript, we use an $\mathrm{ABM}$ framework to 
model and simulate the model nematode $C$. elegans to investigate how chemoattractants affect the movement of worms in both continuous and periodic environments. Within this framework each model C. elegans integrates information about position, heading, and sensing of the external environment to to make movement decisions, resulting in a correlated random walk. Based on their perception of the environment and movement decisions, C. elegans will either locate and move towards the source of the chemoattractant or wander away from the source location. Our chemoattractant is emitted by a strain of engineered bacteria designed to attract and kill nematodes. This strain of bacteria is engineered using the principles of synthetic biology and may serve as a biocontrol mechanism to prevent nematode infections.

Our mathematical modeling is explicitly connected to an experimental setting where we exposed $C$. elegans to various concentrations of the chemoattractant 3-oxohexanoyl-homoserine lactone (3OC6HSL), an Nacyl homoserine lactone (AHL), which is produced by bacteria during the process of quorum sensing (Beale et al., 2006; Fuqua et al., 1994). As such, the results presented herein may be generally tractable to additional scenarios where a small molecule is used by nematodes as a chemoattractant.

\section{BIOLOGICAL BASIS OF OUR MODELING FRAMEWORK}

To model the essential features of the system, we considered several key parameters of both $C$. elegans and a strain of engineered bacteria. Our engineered strain of Escherichia coli secretes the AHL 3OC6HSL. This is readily accomplished by placing the luxI gene, which makes 30C6HSL, downstream of an externally inducible promoter. This allows us to modulate the expression of luxI and thus the amount of 30C6HSL secreted by changing the concentration of an externally applied signaling chemical. The luxI gene has been successfully engineered previously into E. coli (Smith et al., 2014). Our strain of bacteria is minimally motile and thus rests in a fixed position, or colony, within the environment. From this position, the 30C6HSL produced by the bacteria diffuses in a Gaussian fashion (Song et al., 2009). In addition to secreting 3OC6HSL, our engineered bacteria are designed to produce a toxin. Upon ingestion, the engineered bacteria release the toxin, which results in death of the worm. In our system nutrients are not limiting to the ability of the bacteria to produce and secrete $30 \mathrm{OC} 6 \mathrm{HSL}$ or to produce the toxin. We did not explicitly model the growth of the bacteria and assumed that they were initially at carrying capacity within the colony.

Next, we explicitly considered several core behaviors that have been previously described in $C$. elegans when they sense a chemoattractant. In general, C. elegans behavioral response to gradients of chemoattractants involves orientation and movement up the gradient, accumulation, and then habituation. The direction of movement is determined by the lateral motion of the head and is thus a form of klinotaxis (Ward, 1973). Overall movement takes place in two distinct phases: runs, where $C$. elegans moves continuously in a sinusoidal swimming motion, and pirouettes, where $C$. elegans arrests its movement, turns and reorients itself relevant to the attractant gradient. While pirouettes often involve sharp adjustments in orientations, runs are characterized as more or less straight forward motion where the worm may gradually curve to change its orientation within the chemoattractant gradient (Albrecht and Bargmann, 2011). Pirouette frequency has been shown to increase as the worm moves down the concentration gradient (Pierce-Shimomura et al., 1999). Furthermore, pirouettes tend to result in larger reorientations when the worms are farther down the concentration gradient. In contrast, movement speed, turning rate and turning bias remain relatively unchanged when presented with a chemoattractant (Pierce-Shimomura et al., 1999).

While several generalized parameters of $C$. elegans movement have been described in the literature (e.g. (Pierce-Shimomura et al., 1999)), we wanted to examine C. elegans movement within our specific experimental framework to develop a set of parameters that were more specifically matched to our application. To accomplish this, we grew $C$. elegans (strain N2) at room temperature on nematode growth agar medium containing E. coli strain MG1655 as its food source. We placed approximately 100 worms at one end of an M9 agar medium in a $10 \mathrm{~cm}$ diameter Petri dish. At the opposite end, we placed a $10 \mu L$ aliquot of $20 \mu M$ 3OC6HSL. After allowing the worms to become disentangled and hence engage in independent motility (approximately one hour), we performed time-lapse photography at $2.52 \mathrm{X}$ magnification. We then quantified worm length, the time required to complete one sinusoidal swimming motion, the distance travelled per undulation and the turning angle of the worms during a run.

We found no significant correlation between the length of $C$. elegans and forward velocity, forward displacement, and undulation period $\left(R^{2}<0.1, p>0.05\right.$ for each variate). Hence we choose forward velocity as the distinguishing characteristic of each individual worm. We observed an average forward velocity of $0.115 \mathrm{~cm} / \mathrm{sec}$ with a standard deviation of 0.0052 $\mathrm{cm} / \mathrm{sec}$. Here, the minimum velocity observed was $0.0029 \mathrm{~cm} / \mathrm{sec}$ while the maximum velocity observed was $0.0293 \mathrm{~cm} / \mathrm{sec}$. We note that $C$. elegans did not appear to significantly alter their individual forward velocity during a run. Finally, we determined the maximum observed turning angle rate of $44.15^{\circ} / \mathrm{sec}$.

\section{A MODEL THAT DESCRIBES THE INTERACTIONS BETWEEN OUR ENGINEERED BACTERIA AND C. ELEGANS}

Based on these core biological behaviors, we developed a model that describes the interactions between a strain of bacteria engineered to produce a chemoattractant and $C$. elegans. The model contains two kinds of entities or agents, a colony of engineered bacteria and $C$. elegans (or more generically worms). These entities are placed in an environment that represents a circular Petri dish $8 \mathrm{~cm}$ in radius. One bacterial colony is placed in the center of the plate. The colony occupies a circular space $1 \mathrm{~cm}$ in diameter considered to be the capacity size of the colony. 100 worms are randomly placed a distance of $4 \mathrm{~cm}$ from the center of the bacteria colony.

The bacteria are described by two internal state variables; chemoattractant attraction strength $(A)$ and 
diffusion of the chemoattractant in the environment $(D)$. The chemoattractant released by the colony diffuses across the environment creating an isotropic profile of the chemoattractant strength across the medium. The bacteria agent interacts with the worm agents to influence movement decisions through this alteration of the external environment. This concentration is sensed by the worms for movement decisions. The attraction strength, $A$, is effectively a function of the chemoattractant concentration that normalizes the attraction so that $A=1$ is the maximum attraction perceived by a worm.

Each worm stores internal state variables describing their current position $(x, y)$, heading $(\theta)$, and forward velocity $(v)$. Initially, each worm is assigned a random heading and velocity. Headings are drawn from a uniform distribution with a range of $360^{\circ}$. Each worm then selects a forward velocity drawn from a normal distribution with a mean and standard deviation given by the velocity measurements detailed above. If the forward velocity draw exceeds the measured maximum or minimum velocities, the forward velocity is rejected and redrawn.

The simulation consists of two sequential processes at each time step based on the worm's sensing of the environment. These include a reorientation process followed by a displacement process. Through klinotaxis, the worm samples differences in chemoattractant concentration and attempts to reorient itself towards the greatest concentration gradient. With the updated heading, each worm will update its current position by the equations of motion:

$$
\begin{aligned}
\frac{d x_{i}}{d t} & =v_{i} \cos \left(\theta_{i}\right) \\
\frac{d y_{i}}{d t} & =v_{i} \sin \left(\theta_{i}\right)
\end{aligned}
$$

where $i$ indexes the worm. At the end of each step, worms that have landed on the bacteria colony are considered to have ingested the bacteria and died due to the toxin. Worms that have wondered off the edge of the plate are considered to have either not detected or chosen not to detect the chemoattractant and are removed from the population. The simulation is run until every worm either finds the bacterial colony or reaches the edge of the plate. Then, a choice index is computed to determine the proportion of the population that has chosen the bacteria colony:

$$
\text { choice index }=\frac{\text { number worms found bacteria }}{\text { total number worms }} \text {. }
$$

We use a timestep of 1 second for this report.

For the reorientation process, each worm selects a turning angle, $\phi$, based on how strongly the worm perceives the attraction from the chemical gradient $(\rho)$ and the angular displacement between the current heading and the direction towards the bacteria colony $(\Delta \theta)$. We draw displacements from a wrapped Cauchy distribution (WCD) that is limited to a maximum absolute displacement $\phi_{M}$. We use this distribution as it is parameterized by a preferred angle and a shape parameter that reflects the strength of the attraction from the chemical gradient. Additionally, the inverse cumulative density function for the unconstrained WCD can be given in closed form to simplify the probability density sampling process
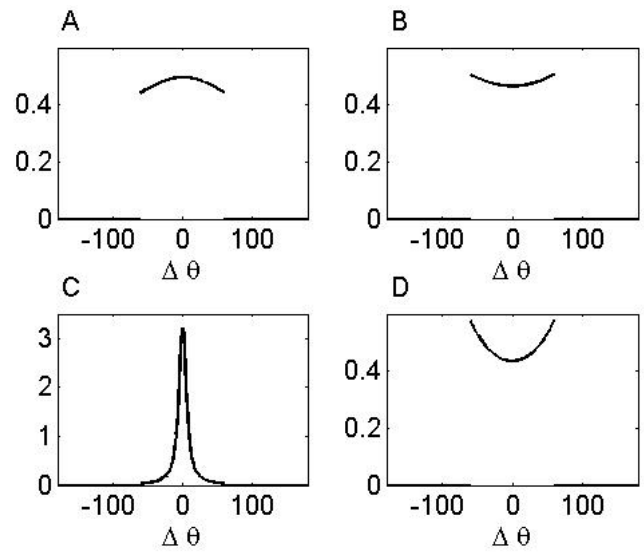

Fig. 1. Turning angle distribution, $f(\phi ; \rho, \Delta \theta)$ : Examples of the turning angle distribution for different parameterizations, A: $\rho=$ $0.1, \Delta \theta=0^{\circ} ; \mathrm{B}: \rho=0.1, \Delta \theta=180^{\circ} ; \mathrm{C}: \rho=0.9, \Delta \theta=0^{\circ}$; D $\rho=0.9, \Delta \theta=180^{\circ} . \phi_{M}=60^{\circ}$ in all panels.

(Haefner, 2005). The probability density function for this constrained WCD is defined as:

$$
f(\phi ; \rho, \Delta \theta)= \begin{cases}\frac{1}{Z} f_{W C D}(\phi ; \rho, \Delta \theta) & \text { if }|\phi|<\phi_{M} \\ 0 & \text { otherwise }\end{cases}
$$

where $Z$ is a normalization factor for the probability density function, $f_{W C D}(\phi ; \rho, \Delta \theta)$ is the WCD probability density function, and $0 \leq \rho \leq 1$. When $\rho=0$ turning angles are drawn from a uniform distribution and when $\rho=1$ the turning angle is drawn from a Dirac $\delta$-function distribution representing a ballistic motion. Values of $\rho$ between this range are interpolating between these two extremes. While we measured a maximum turning rate of $44.15^{\circ} / \mathrm{sec}$ exclusive of pirouettes, we set $\phi_{M}=60^{\circ}$ to allow for conceptualization of pirouette type motion. Given our 1 second time step, this allows for a full pirouette to occur in the approximate time period that we observe full pirouette motion.

Figure 1 shows examples of this turning angle distribution for parameters describing different chemoattractant gradients sensed by the worms and relative headings of the worms to the chemoattractant gradient. Panels A and B illustrate cases where there is a weakly sensed concentration $(\rho=0.1)$ and choice of turning angle is nearly uniform. The worms current heading is towards or away from the chemoattractant in panels $\mathrm{A}$ or $\mathrm{B}$, respectively. In Panels $\mathrm{C}$ and $\mathrm{D}$, the sensed chemoattractant gradient is very strong $(\rho=0.9)$ and the turning angle is strongly biased. In panel $\mathrm{C}$ the worm is headed towards the colony and will have very little variation in its heading. In panel D the worm is headed away from the colony and is likely to make a large turn. We conceptualize a pirouette as a sequence of such large turns to reorient itself amongst the chemoattractant gradient.

Each worm recomputes its individual $\rho$ value at each time step based on the chemoattractant concentration at its current location. In standard chemotaxis assays, this concentration is time independent and decreasing exponentially consistent with a diffusion process (Ward, 1973). Hence, $\rho$ is described by

$$
\rho(x, y)=A e^{\frac{-\left(x^{2}+y^{2}\right)}{2 D^{2}}} .
$$




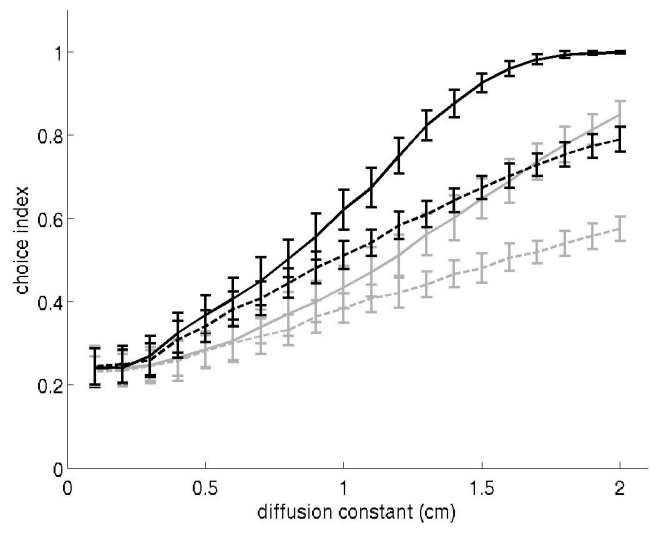

Fig. 2. Choice index as a function of diffusion constant in static gradient: Comparison of the impact of $D$ on the choice index for $A=0.1$ (grey line), $A=0.9$ (black line). Without learning (solid lines) the choice index will asymptote to its maximum value of 1 for both cases; however, this will happen more rapidly for greater $A$. Learning (dashed lines) can significantly reduce the choice index for cases where the choice index exceeds 0.40 without learning. Error bars represent one standard deviation over 100 trials.

We set a global parameter $\rho_{\max }=0.9$ that sets the maximum value of $\rho$ in order to avoid cases of pure ballistic motion.

This model was implemented in the NetLogo programming language and all simulations were performed within the NetLogo environment (Wilensky, 1999). NetLogo is well designed for models such as this one where agent behavior is dominated by local interactions over a short period of time and agents are mobile and act concurrently on a grid space (Railsback et al., 2006).

\section{DIFFUSION CONSTANT OF AHL IS A CRITICAL PARAMETER THAT DETERMINES CHOICE}

Initially, we used the model to explore the impact of diffusion constant $(D)$ and attraction strength $(A)$ of chemoattractants produced by our engineered bacteria in guiding the chemotaxis of the worms. To accomplish this, we determined the choice index of the worms while varying the attraction strength and diffusion constant of AHL. Experimentally, these parameters may be perturbed by changing the rate or type of AHL produced from the engineered bacteria or by changing the media components in which the engineered bacteria and worms are grown. We performed 100 simulations of each choice of parameter pairs $A$ and $D$.

When there is no AHL, or very high concentrations of AHL within which the worms cannot determine a chemoattractant gradient $(A=0)$, the worms engage in purely random turning decisions limited only by the maximum turn angle $\phi_{M}$ resulting in a mean choice index of 0.23 . This indicates that, on average, only $23 \%$ of the worms will find the bacterial colony before leaving the plate. For non-zero choices of $A$ we find the choice index of the worm indistinguishable from the $A=0$ case for $D \leq 0.3 \mathrm{~cm}$ as shown in figure 2 . Once $D$ exceeds this value, the choice index becomes an approximately linear function of $D$ for any choice of $A$ until it approaches the choice index of 1 resulting

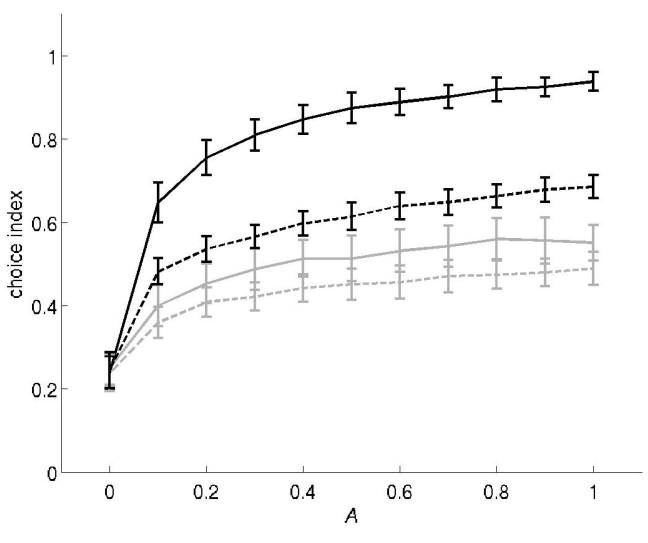

Fig. 3. Choice index as function of attraction strength in static gradient: Comparison of impact of $A$ on the choice index for $D=0.9 \mathrm{~cm}$ (grey lines) and $D=1.5 \mathrm{~cm}$ (black line). Without learning (solid lines), in both cases there is an initially large effect from increasing the strength of AHL that quickly asymptotes. Learning (dashed lines) makes a marked decrease in choice index. The choice index is greater for greater diffusion constants $D$. Error bars represent one standard deviation over 100 trials.

in an overall sigmoidal shape. We also observe from figure 2 that for larger choices of $A$ the choice index will reach this peak for smaller values of $D$. Thus in medium that allows for greater diffusion of the chemoattractant, a smaller attraction strength or equivalently AHL concentration production may be required to attract the worms to the bacterial colony. While increasing the diffusion constant so that the chemoattractant is detectable across the entire landscape may be sufficient to attract all worms towards the bacteria colony, figure 3 indicates that for a given value of $D$, we may not be able to produce sufficient chemoattractant to attract all the worms in the environment. That is, for a diffusion constant of $0.9 \mathrm{~cm}$ we may only attract half the worms in the environment regardless of the attraction strength of the AHL. However a small increase to $1.5 \mathrm{~cm}$ may permit for a sufficiently large proportion of the worms to be attracted to the colony even at low AHL concentration levels.

These modeling predictions indicate that choice index is sensitive to the diffusion constant of AHL. As the diffusion constant is increased, the time required for AHL to begin drawing the worms towards the engineered bacteria decreases. As such, fewer worms are removed from the system having not found the engineered bacteria. Conversely, when the diffusion constant is sufficiently low, the AHL does not reach the worms at a sufficiently fast time scale, and worms are removed from the system before they can locate the engineered bacteria. For a given diffusion constant, large increases in attraction strength appear to play a minor role in increasing choice index. Even with low attraction strength, once the AHL reaches the worms (controlled via diffusion constant) they re-orient their movement towards the engineered bacteria and will eventually locate the colony. We verified this observation of sensitivity by computing the relative sensitivity of the choice index $(C I)$ to each 


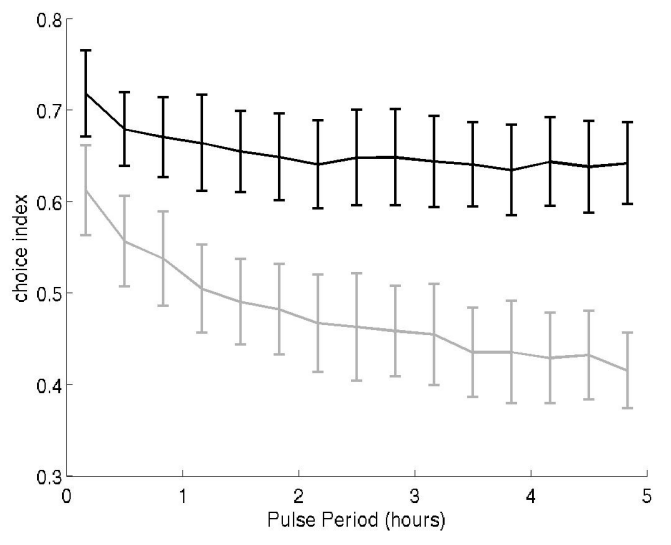

Fig. 4. Choice index as a function of pulse period for a pulsing chemoattractant source: Comparison of the impact of pulse period on the choice index for $A=0.1$ (grey line), $A=1$ (black line). For high levels of attraction strength, there is little sensitivity of the choice index to the pulse period with choice index remaining approximately constant after a 1 hour pulse period. However at low levels of AHL attractiveness pulse period is a critical factor in determining the choice index. Error bars represent one standard deviation over 100 trials.

parameter $(p)$,

$$
\text { relative sensitivity }=\frac{\Delta C I / C I}{\Delta p / p} .
$$

While the relative sensitivity to $A$ is nearly constant, prior to nearing the asymptote, the relative sensitivity to $D$ can be 6 to 8 times larger than that for $A$.

\section{REDUCTION IN CHOICE INDEX DUE TO LEARNING IS GREATER AT HIGHER DIFFUSION CONSTANTS}

Previous studies have indicated that $C$. elegans will learn and preferentially avoid bacteria that harm it (Zhang et al., 2005). Under these conditions, C. elegans will often learn to avoid the chemoattractant secreted by the toxic bacteria (Beale et al., 2006). While a quantitative framework that examines learning has yet to be established, we included this parameter in our equations as it is likely to affect chemoattraction.

Our model includes an internalized learning state variable for each worm. The mechanism we employed is one where the worm becomes less biased towards the bacterial colony as the worm senses an increased choice index. We implemented this by rescaling the attraction strength used for the computation of $\rho$,

$$
A_{e f f}=\left(1-\frac{C I^{5}}{.25^{5}+C I^{5}}\right) A .
$$

We previously noted that in the absence of a chemoattractant, the choice index will be 0.23 . In our learning model, the bias of the worms towards the bacteria colony will make a sharp transition around this choice index. We observe that in learning scenarios, the choice index tends to threshold around 0.5 . For the choice of learning model we use $A_{e f f}$, which will be very small when the choice index is 0.5 .

Using our modified model, we explored how the introduction of learning affected choice index when diffusion constant and attraction strength were varied.

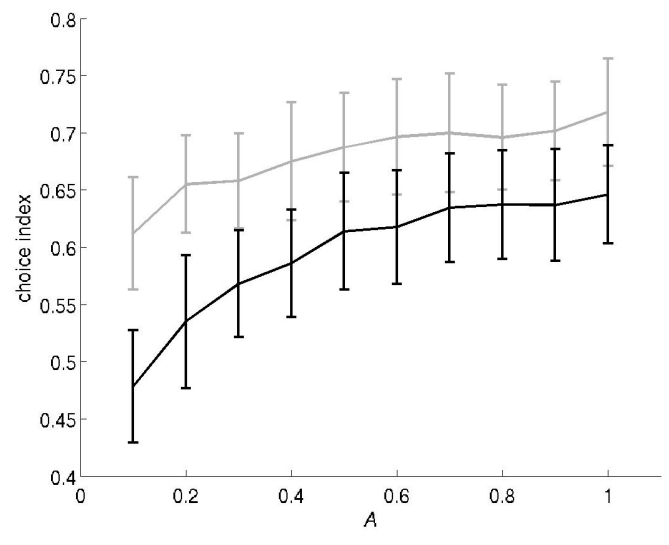

Fig. 5. Choice index as function of attraction strength from a pulsing chemoattractant source: Comparison of impact of $A$ on the choice index for pulse period of 10 minutes (grey line) and 1 hour (black line). At shorter pulse periods, there is relatively little sensitivity to the attraction strength. However, at long pulse periods the AHL attractiveness is a critical factor for determining choice index. Error bars represent one standard deviation over 100 trials.

Our model predicts that the introduction of learning serves to reduce the choice index, on average, for each diffusion constant (Figure 2) and attraction strength (Figure 3) examined. However, Figure 2 indicates that, if the diffusion constant is low, and for both attraction strengths examined, there exists less difference between the two cases where learning is and is not included. As the diffusion constant is increased, the disparity between the modeling prediction with and without learning becomes greater.

This trend is echoed in Figure 2. When the diffusion constant is large, there is a greater difference in the choice index predicted for when the model includes, or does not include, learning. This difference increases as attraction strength increases. However, when the diffusion constant is small, the difference in choice index between the two models is minimal for all values of attraction strength examined.

Overall, the inclusion of learning in the model serves to reduce the positive relationship between diffusion constant and choice index. Our modeling results suggest that the interplay between the diffusion rate of a chemoattractant and learning of the worm must be carefully considered to maximize choice in an experimental setting.

\section{ATTRACTION STRENGTH DRIVES CHOICE IN C. ELEGANS WHEN THE CHEMOATTRACTANT IS PULSED}

To date, the majority of studies on the effect of chemoattractants on motion and behavior of $C$. elegans have been performed in static environments, wherein the chemoattractant is always present and forms a time independent concentration gradient. However, in practice, few natural environments are static, and chemoattractant concentrations are likely to fluctuate over time. These dynamic environments may have a significant influence on the behavior of $C$. elegans. For example, it has recently been demonstrated $C$. elegans behavior is affected by periodically pulsing of a chemoattractant that 
is distributed throughout the environment (Albrecht and Bargmann, 2011). However, it remains unclear as to how the properties of a chemoattractant affect choice when the attractant is periodically pulsed.

To examine how periodic pulses of AHL emitted by the bacterial colony might impact the choice index of the worms, we modified our model to include an internal state variable $(P)$ representing the pulse period of secretion of the chemoattractant by the bacterial colony in the description of the bacteria agent. Specifically, the external environmental influence on the worms' perception of the attraction strength $(\rho)$ will be both space and time dependent. We idealize the bacteria colony to make periodic instantaneous releases of chemoattractant from the center of the colony. The concentration of chemoattractant in the environment $(u(x, y, t))$ is then given by a superposition of solutions of the Cauchy problem for the 2-dimensional diffusion equation:

$$
\begin{cases}u_{t}=\nabla u & \text { in } \mathbb{R}^{2} \times(0, \infty) \\ u(\cdot, 0)=g & \text { on } \mathbb{R}^{2}\end{cases}
$$

where $g(x, y)$ represents one pulse of chemoattractant. That is, $g(x, y)$ has the properties:

$$
\begin{aligned}
g(x, y) & =0 \text { for all }(x, y) \neq(0,0) \\
\int_{\mathbb{R}^{2}} g(x) d x & =A
\end{aligned}
$$

The sensed attraction strength $(\rho)$ is then given by a normalization of the fundamental solution of the diffusion equation in 2-dimensions:

$$
\rho(x, y, t)=\sum_{k=1}^{n(t)} \frac{A * N}{4 \pi D *\left(t-\tau_{k}\right)} e^{\frac{-\left(x^{2}+y^{2}\right)}{4 D *\left(t-\tau_{k}\right)}}
$$

where $\tau_{k}$ are the pulse times up to time $t$ and $N=\frac{1}{4} \pi e$ is a normalization to make $\rho=A$ at the boundary of the $1 \mathrm{~cm}$ radius bacteria colony. Experimentally, AHL pulsing may be realized by introducing and removing the chemical that induces luxI expression within the experimental setup. We use previous estimates of the diffusion constant for 30C6HSL in a $0.2 \% \mathrm{M} 9$ agar of $D=0.4 \mathrm{~cm}^{2} / \mathrm{hr}$ (Song et al., 2009). At any location where $\rho$ exceeds the previously defined $\rho_{\max }$, the value of $\rho$ will be set to this $\rho_{\max }$.

Figure 4 examines the influence of the pulsing period for the chemoattractant emitted by the bacterial colony for different levels of the attraction strength of the chemoattractant. First, we note that with a pulsing chemoattractant source, choice index does not reach 1. That is, we cannot attract all the worms to the bacterial colony. As such, overall pulsing the attractant reduces the ability of the engineered bacteria to attract the worms. Our model predicts that increasing pulse period reduces choice index. This is due to the fact that at longer pulse periods, the concentration of AHL in the environment is lower as compared to shorter pulse periods. We note that the reduction in choice index as pulse period is increased in markedly reduced when the attraction strength is sufficient high. This may create a significant trade off between the attraction strength of the emitted chemoattractant and the pulsing period. On the one hand, to attract a significant number of worms using an AHL with a low attraction strength, a relatively high rate of pulsing may be required. This pulsing rate may be impractical in the engineered bacteria. On the other hand, when the AHL is pulsed at longer pulsing periods, which may be more feasible in engineered bacteria, an AHL with a significantly high attraction strength (or concentration) may be required.

Figure 5 examines the influence of the chemoattractant attraction strength for different pulse periods. Our model predicts that, even at low attraction strength and long pulse periods, the choice index is significantly different than when there is no AHL present $(A=0$, $\mathrm{CI}=0.23$ ). Furthermore, our model predicts that at long pulse periods, attraction strength has a greater impact on the choice index, relative to shorter pulse periods. These results echo conclusions drawn from Figure 4; choosing an AHL with a high attraction strength is critical when the chemoattractant is pulsed in the environment.

\section{DISCUSSION}

This manuscript describes an ABM framework to explore how the properties of a chemoattractant influence choice in nematodes, such as C. elegans. The parameters of our model are connected to measurements made when C. elegans was presented with an AHL that can be readily secreted from engineered bacteria. While the specific setup studied here arguably does not require the ABM framework utilized, the present study provides as an additional outcome a proof of concept for utilizing an ABM framework in this application context. This study provides preliminary results, and a modeling and simulation framework for future studies integrating a higher degree of individual autonomy between worm agents including individual lifecycles and adaptive worm agent motion decisions based on learning and memory from a more limited interaction with other worm agents.

Using this modeling framework we have uncovered several tradeoffs that may influence how $C$. elegans perceives chemoattractants. Specifically, we have observed that when AHL is emitted continuously from the engineered bacteria, selecting an AHL that diffuses quickly will lead to the greatest choice index. Interestingly, nematodes are known to be attracted to a variety of AHLs and additional chemicals, all of which likely have different diffusion rates (Zhang et al., 2005; Beale et al., 2006). However, our model cautions against using this principle when the chemoattractant is emitted from a toxic bacteria. If the worm learns to associate the chemoattractant with injury, selecting a chemoattractant with a slower diffusion rate would be preferable. While we have modeled learning as a function of choice index, it will be interesting the future to develop a more quantitative, experimentally driven understanding of learning, as well as memory, so as to further refine the implementation of this critical facet of the model.

Our framework has also examined choice index when the chemoattractant is pulsed. In the laboratory setting, this may reflect dynamic regulation of chemoattractant producing genes in engineered bacteria. In the natural environment, this may represent a population of bacteria or other organisms whose density, and thus ability to secrete chemoattractants, changes over time. While we have yet to examine how learning impacts choice index under this dynamics condition, it is interesting to hypothesize that dynamic regulation of the chemoattractant can 
reduce learning and memory, and thus serve to increase choice index.

The principles and tradeoffs observed in this study may be useful in developing a more profound understanding of how nematodes integrate and respond to signals in their environment. Furthermore, they may serve as design principles to allow optimization of bacteria specifically engineered to kill parasitic nematodes.

\section{ACKNOWLEDGEMENTS}

This research is supported by a Presidents Faculty Research and Development Grant \#335318 through Nova Southeastern University and a HBCU/MI Equipment/Instrumentation from the Department of Defense/Army Research Office (W911NF-14-1-0070). We would like to thanks Olena Bracho and Cyril Manchery for providing unpublished data and discussions on the experimental framework.

\section{REFERENCES}

Albrecht, D. R. and Bargmann, C. I. (2011). Highcontent behavioral analysis of caenorhabditis elegans in precise spatiotemporal chemical environments. Nature methods, 8(7):599-605.

Beale, E., Li, G., Tan, M.-W., and Rumbaugh, K. P. (2006). Caenorhabditis elegans senses bacterial autoinducers. Applied and environmental microbiology, 72(7):5135-5137.

Brooker, S., Bethony, J., and Hotez, P. J. (2004). Human hookworm infection in the 21 st century. Advances in parasitology, 58:197-288.

Chaisson, K. E. and Hallem, E. A. (2012). Chemosensory behaviors of parasites. Trends in parasitology, 28(10):427-436.

De Silva, N. R., Brooker, S., Hotez, P. J., Montresor, A., Engels, D., and Savioli, L. (2003). Soiltransmitted helminth infections: updating the global picture. Trends in parasitology, 19(12):547-551.

Fuqua, W. C., Winans, S. C., and Greenberg, E. P. (1994). Quorum sensing in bacteria: the luxr-luxi family of cell density-responsive transcriptional regulators. Journal of bacteriology, 176(2):269.

Grabe, K. and Haas, W. (2004). Navigation within host tissues: Schistosoma mansoni and trichobilharzia ocellata schistosomula respond to chemical gradients. International journal for parasitology, 34(8):927-934.

Haas, W. (2003). Parasitic worms: strategies of host finding, recognition and invasion. Zoology, 106(4):349364.

Haefner, J. W. (2005). Modeling Biological Systems:: Principles and Applications. Springer Science \& Business Media.

Hotez, P. J., Brindley, P. J., Bethony, J. M., King, C. H., Pearce, E. J., Jacobson, J., et al. (2008). Helminth infections: the great neglected tropical diseases. The Journal of clinical investigation, 118(4):1311-1321.

Lafferty, K. (1999). The evolution of trophic transmission. Parasitology Today, 15(3):111-115.

Nathan, R. (2008). An emerging movement ecology paradigm. Proceedings of the National Academy of Sciences, 105(49):19050-19051.
Pierce-Shimomura, J. T., Morse, T. M., and Lockery, S. R. (1999). The fundamental role of pirouettes in caenorhabditis elegans chemotaxis. The journal of neuroscience, 19(21):9557-9569.

Rae, R. G., Robertson, J. F., and Wilson, M. J. (2009). Chemoattraction and host preference of the gastropod parasitic nematode phasmarhabditis hermaphrodita. Journal of parasitology, 95(3):517526.

Railsback, S. F. and Grimm, V. (2011). Agent-based and individual-based modeling: a practical introduction. Princeton university press.

Railsback, S. F., Lytinen, S. L., and Jackson, S. K. (2006). Agent-based simulation platforms: Review and development recommendations. Simulation, 82(9):609-623.

Safer, D., Brenes, M., Dunipace, S., and Schad, G. (2007). Urocanic acid is a major chemoattractant for the skin-penetrating parasitic nematode strongyloides stercoralis. Proceedings of the National Academy of Sciences, 104(5):1627-1630.

Sciacca, J., Forbes, W. M., Ashton, F. T., Lombardini, E., Gamble, H. R., and Schad, G. A. (2002). Response to carbon dioxide by the infective larvae of three species of parasitic nematodes. Parasitology international, 51(1):53-62.

Smith, R., Tan, C., Srimani, J. K., Pai, A., Riccione, K. A., Song, H., and You, L. (2014). Programmed allee effect in bacteria causes a tradeoff between population spread and survival. Proceedings of the National Academy of Sciences, 111(5):1969-1974.

Song, H., Payne, S., Gray, M., and You, L. (2009). Spatiotemporal modulation of biodiversity in a synthetic chemical-mediated ecosystem. Nature chemical biology, 5(12):929-935.

Tang, W. and Bennett, D. A. (2010). Agent-based modeling of animal movement: A review. Geography Compass, 4(7):682-700.

Ward, S. (1973). Chemotaxis by the nematode caenorhabditis elegans: identification of attractants and analysis of the response by use of mutants. Proceedings of the National Academy of Sciences, 70(3):817-821.

Wilensky, U. (1999). Netlogo, center for connected learning and computer-based modeling, northwestern university. http://ccl.northwestern.edu/netlogo/.

Zhang, Y., Lu, H., and Bargmann, C. I. (2005). Pathogenic bacteria induce aversive olfactory learning in caenorhabditis elegans. Nature, 438(7065):179-184.

Zuckerman, B. M. and Jansson, H. (1984). Nematode chemotaxis and possible mechanisms of host/prey recognition. Annual Review of Phytopathology, 22(1):95-113. 